\title{
Numerical Simulation for Time Dependent Materials
}

\author{
WX Zhang ${ }^{1, *}$ \\ ${ }^{1}$ Nantong Polytechic College, Nantong, Jiangsu, 226002, China
}

\begin{abstract}
Time related materials have the advantages of high strength and easy processing. As structural materials, they are more and more widely used in chemical industry, machinery, aerospace, architecture and marine development and other industrial fields. The static and dynamic analysis of these materials and structures under various loading and service conditions has become an important research content in the field of mechanics. Based on the traditional method, this paper presents a simulation technique suitable for solving time-dependent material and structure problems, and gives the treatment method of boundary conditions.
\end{abstract}

\section{Introduction}

With the rapid development of science and technology, many new materials, such as various types of polymer, synthetic rubber, paint, glass, ceramics and so on, have been widely used in modern industry. These materials not only have the characteristics of elastic solid, but also have the characteristics of viscous fluid, that is, timedependent materials. Such materials usually have creep and relaxation characteristics. The creep of rock under geological conditions can produce considerable deformation, but the required stress is not necessarily very large [1-3]. Stress relaxation refers to the phenomenon that when the total strain of viscoelastic material is constant, due to the increasing of viscous strain component in the sample, the rebound strain component gradually decreases with time, resulting in the gradual decrease of deformation recovery force.
Stress relaxation curve is an important basis for determining Poisson's ratio of materials [4, 5].

In reality, we often encounter the mechanical problems of structural materials working under extreme conditions such as high temperature, high pressure and high speed, such as the analysis of severe brittle fracture and collapse accidents of some structures and devices. However, these accidents do not occur at the moment of loading, but suddenly after a period of time, which we call "delayed fracture". Because of its "delay" and "sudden" characteristics, like fatigue damage, it is difficult for people to prevent. Therefore, the study of the "time effect" of materials, especially the creep characteristics at high temperature, has become a hot topic in practical engineering.

\section{Solution method}

The integral constitutive relation of isotropic viscoelastic materials is

$$
\begin{aligned}
& \sigma_{i j}+\xi \frac{\partial \sigma_{i j}}{\partial t}=2 \eta e_{i j}+2 \frac{\partial \varepsilon_{i j}}{\partial t} \\
& \sigma=2 \kappa e
\end{aligned}
$$

where

$$
\begin{aligned}
& \varepsilon_{i j}=\frac{1}{2}\left(u_{i, j}+u_{j, i}\right) \\
& \sigma_{i j, j}+g_{i}=0
\end{aligned}
$$

The elastic modulus satisfies the following relationship

$$
E \otimes f=\int_{0}^{t} E(t-\chi) \frac{\partial f(\chi)}{\partial \chi} d \chi
$$


The relaxation modulus can be described in series as follows:

$$
E(t)=E_{0}+\sum_{g} \int_{0}^{t} E_{g} \exp \left(-c_{g} t\right)
$$

The relationship between shear stress and transverse shear strain satisfies the convolution equation

$$
\begin{aligned}
& \varepsilon_{1}=s_{11} \otimes \sigma_{1}+s_{12} \otimes \sigma_{2} \\
& \varepsilon_{2}=s_{12} \otimes \sigma_{1}+s_{22} \otimes \sigma_{2} \\
& \varepsilon_{3}=s_{33} \otimes \sigma_{3}, \varepsilon_{4}=s_{44} \otimes \sigma_{4} \\
& \varepsilon_{5}=s_{44} \otimes \sigma_{4}, \varepsilon_{6}=s_{66} \otimes \sigma_{6}
\end{aligned}
$$

Substituting Eq. (4) into Eq. (5), we have

$$
\begin{aligned}
& N_{x}=\frac{h}{1-v} E \otimes\left(\varepsilon_{x}+v \varepsilon_{y}\right) \\
& N_{y}=\frac{h}{1-v} E \otimes\left(v \varepsilon_{x}+\varepsilon_{y}\right) \\
& N_{x y}=\frac{h}{1-v} E \otimes \gamma_{x y} \\
& M_{x}=\frac{h^{2}}{12(1-v)} E \otimes w_{x}
\end{aligned}
$$

and

$$
\begin{aligned}
& E \otimes \gamma_{x y}=\frac{2(1+v)}{h} N_{x y} \\
& M_{y}=\frac{h^{2}}{12(1-v)} E \otimes w_{y}
\end{aligned}
$$

The coefficient satisfies the following relation

$$
\left[A_{i j}\right]=\left[\begin{array}{lll}
\beta_{11}(b) & \beta_{12}(b) & \beta_{13}(b) \\
\beta_{21}(b) & \beta_{22}(b) & \beta_{23}(b) \\
\beta_{31}(b) & \beta_{32}(b) & \beta_{33}(b) \\
\beta_{11}(a) & \beta_{12}(a) & \beta_{13}(a) \\
\beta_{21}(a) & \beta_{22}(a) & \beta_{23}(a)
\end{array}\right]
$$

After simplification, we get

$$
\left|A_{i j}\right|=\left|\begin{array}{ll}
J_{2}(\mu b) & Y_{2}(\mu b) \\
J_{2}(\mu a) & Y_{2}(\mu a)
\end{array}\right| \otimes\left|\begin{array}{llll}
\alpha_{11}(b) & \alpha_{12}(b) & \beta_{11}(b) & \beta_{12}(b) \\
\alpha_{21}(b) & \alpha_{22}(b) & \beta_{21}(b) & \beta_{22}(b) \\
\alpha_{11}(a) & \alpha_{12}(a) & \beta_{11}(a) & \beta_{12}(a) \\
\alpha_{21}(a) & \alpha_{22}(a) & \beta_{21}(a) & \beta_{12}(a)
\end{array}\right|
$$




\section{Numerical example}

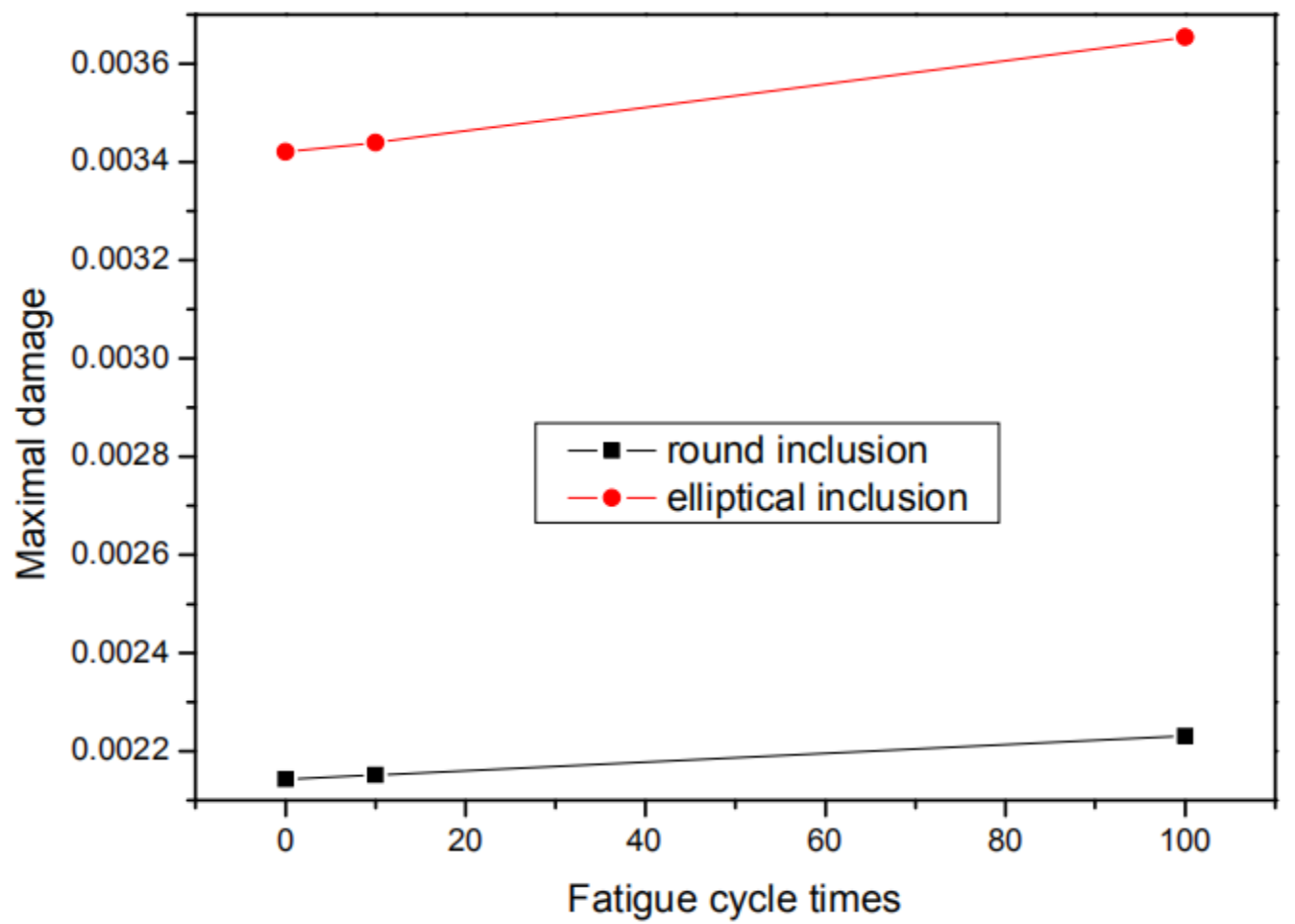

Figure 1. Displacement versus time curves

In this section, in order to verify the correctness of the above method, we have carried out numerical simulation for Maxwell solid model. The curve of displacement versus time is given in Fig. 1, and the results are in good agreement with the actual situation.

\section{Conclusion}

Creep and stress relaxation are essentially the same. Stress relaxation can be regarded as "multistage" creep with decreasing stress. Materials with high creep resistance have higher resistance to stress relaxation. However, it is difficult to use creep data to estimate stress relaxation data. Some materials exhibit very slow stress relaxation even at room temperature, especially at high temperatures.

\section{Reference}

1. Berdichevsky, V.L., Le, K.C. (2007) Dislocation nucleation and work hardening in anti-planed constrained shear. Contin. Mech. Thermodyn., 18: 455-467.

2. Gao, C.F., Wang, M.Z. (2000) Collinear permeable cracks between dissimilar piezoelectric materials. Int. J. Solids Struct., 37: 4949-4967.

3. Gruebner, O., Kamlah, M., Munz, D. (2003) Finite element analysis of cracks in piezoelectric materials taking into account the permittivity of crack medium. Eng. Fract. Mech., 70: 1399-1413.

4. Faghidian, S.A. (2016) Unified formulation of the stress field of saint-Venant's flexure problem for symmetric cross-sections. Int. J. Mech. Sci., 111-112: 65-72.

5. Pipkin, A.C., Rogers, T.G. (1968) A nonlinear integral representation for viscoelastic behavior. J. Mech. Phys. Solids, 16: 59-72. 